\title{
Why big pharma needs to learn the three 'R's
}

Small companies continue to capitalize in finding alternative uses or improved versions of drugs originating from large companies.

David Bradley

Repositioning, reprofiling, repurposing. Whatever you call it, finding new careers for old drugs is fast becoming big business.

Considerable revenues and savings are to there to be made in discovering alternative purposes for known compounds.

As Nobel laureate James Black famously said, "The most fruitful basis of the discovery of a new drug is to start with an old drug." With faith dwindling in new technologies to drive the drug discovery engine, the need for scientific approaches to cultivating new uses for existing drugs, or those that are about to enter the market, is rising in importance.

Small wonder then that companies have sprouted to fill the void. At least a dozen compounds are now in a strong new position and smaller companies are working on countless compounds.

Although big pharma is interested in obtaining additional indications for their existing compounds, companies seem reluctant to spin out separate business divisions to maximize uses for their products. Repositioning ideas are sped through the process from an internal champion, serendipitous observation, or from outside companies with proprietary insights.

"Repositioning requires a substantially multidisciplinary approach, which big pharma is inefficient in generating," says David Cavalla, who heads Cambridge-based Arachnova, a mid-stage development company based on therapeutic switches.

This is in spite of many success stories of expanded uses for drugs resulting from experimental observations - Pfizer's sildenafil (Viagra) in erectile dysfunction, Lilly's duloxetine (Cymbalta) in stress urinary incontinence and Celgene's thalidomide (Thalomid) in severe erythema nodosum leprosum are classic examples of drugs that ended up being used in entirely unexpected ways.

Potential new disease indications for, or improved versions of, existing drugs are cropping up in unlikely situations. A group led by David Gutmann of Washington University School of Medicine in St Louis, USA, has shown that the immunosuppressant rapamycin could help treat childhood brain tumours. Enzo Bonora of the University of Verona, Italy, has shown that imatinib (Gleevec; Novartis), for chronic myeloid leukaemia also has activity in type 2 diabetes. And, ironically, given its origins, Rakesh Kukreja of the Virginia Commonwealth University, USA, has demonstrated that Viagra can also reduce heart damage in patients on doxorubicin chemotherapy for breast cancer, leukaemia and sarcomas.

Pursuing such strategies would make commercial sense for big pharma. Starting with a known clinical history for a compound shortcuts much of the early testing and clinical trials, dramatically reducing development times and costs, says Christopher Lipinski, who consults for indications discovery company Melior Discovery. "Companies can leverage value out of their compounds without exposing themselves to new risks, as the upfront costs of preliminary testing clinical trials have already been done," he says.

One disincentive for big pharma is that an initial programme could be hampered by a problem in a repositioning programme. "Companies usually hold off on such efforts until the candidate gets approval for its initial indication or development for its initial indication has been discontinued," says Ted Ashburn, Senior Director of Business Development at Dynogen Pharmaceuticals, a biopharmaceutical company developing reprofiled drugs for genitourinary and gastrointestinal disorders.

Repositioning is not without its challenges, says Ashburn. "For instance, the original data package may not meet current regulatory standards, intellectual property issues can be complex, and gaining access to a positioning candidate's patent estate and data package can be difficult," he says.

So large pharmaceutical companies will begin to focus more on repositioning programmes only if ideas for repositioning are more attractive from an economic standpoint than their best ideas for original drug development programmes. The problem, however, lies in knowing which potential directions any one compound could go in and which to follow.

This inertia of big companies partly explains why smaller companies and outsiders can steal a march and come up with many of the repositioning ideas. Nevertheless, it is in big pharma's interest to devote more resources to its existing portfolios. Reprofiling will be become an increasing focus, rivaling drug discovery based on new chemical entities, for big and small companies over the next 10 years, predicts Cavalla. 